\title{
Métodos de avaliação de massa de forragem em pastagens de capim-estrela submetidas a intensidades de pastejo
}

\author{
Estimate methods of the herbage mass on stargrass submitted to grazing intensities \\ Domenico Sales Rocha Arruda ${ }^{\mathrm{I}}$ Marcos Weber do Canto ${ }^{\mathrm{II}}$ Clóves Cabreira Jobim ${ }^{\mathrm{II}}$ \\ Paulo César de Faccio Carvalho ${ }^{\text {III }}$
}

\section{RESUMO}

\begin{abstract}
O experimento foi conduzido para comparar a eficiência dos métodos do disco medidor de forragem (DMF), o bastão graduado (BG), a régua e o medidor de capacitância (MC), utilizados em estimativas da massa de forragem (MF) em pastagens. Os instrumentos foram empregados em pastagens de capim-estrela (Cynodon nlemfuensis Vanderyst var. nlemfuensis) usadas sob condições de pastejo contínuo, taxa de lotação variável e em diferentes alturas do pasto. $O$ delineamento foi o inteiramente ao acaso com duas repetições $e$ os tratamentos foram quatro alturas do pasto: $5,10,15 e$ $20 \mathrm{~cm}$. A altura do pasto nos piquetes foi mantida por ajustes da taxa de lotação, usando-se novilhas da raça Nelore (Bos indicus). As avaliações nos pastos foram realizadas em períodos mensais, de 7/11/2007 a 26/4/2008. A efetividade de cada método para quantificar a MF foi avaliada por equação de regressão. O MC não apresentou resultados consistentes. Os valores obtidos de coeficiente de determinação para avaliação da MF foram baixos e inferiores a 0,63 com o BG, DMF e com a régua. Medições de MF em pastos de capim-estrela sob pastejo contínuo podem ser realizadas preferivelmente com o $D M F$, ои com o $B G$ e a régua.
\end{abstract}

Palavras-chave: altura do pasto, bastão graduado, Cynodon nlemfuensis, disco medidor de forragem, medidor de capacitância, régua.

\section{ABSTRACT}

The experiment was conducted aiming to compare the efficiency of the methods rising plate meter (RP), sward stick (SS), ruler and capacitance meter (CM), used to estimate herbage mass on pastures. The instruments were used in stargrass pasture (Cynodon nlemfuensis Vanderyst var. nlemfuensis) under continuous stocking, variable stocking rate, and sward heights. The design was completely randomized with two replicates and the treatments were sward heights: 5, 10,15 , and $20 \mathrm{~cm}$. The sward height at paddock was maintained by stocking rate adjustments using Nellore heifers (Bos indicus). The swards were evaluated monthly, from November $7^{\text {th }}, 2007$ to April $26^{\text {th }}$, 2008. The effectiveness of each method to quantify the herbage mass was evaluated by the regression equation. The capacitance meter did not show consistent results. The values of coefficient of determination for the evaluation of herbage mass were low and less than 0.63 with the SS, RP and the ruler. Measurements of herbage mass on swards of stargrass under continuous stocking can be performed preferably with the RP, or with the SS and the ruler.

Key words: capacitance meter, Cynodon nlemfuensis, rising plate meter, sward height, sward stick, ruler.

\section{INTRODUÇÃO}

A massa de forragem (MF) é definida como a quantidade de matéria seca (MS) presente instantaneamente acima do nível do solo por unidade de área (HODGSON, 1979; FGTC, 1992), sendo usualmente expressa em $\mathrm{kg} \mathrm{ha}^{-1}$ de MS. BENKOBI et al. (2000), BRAGA et al. (2009) e PELLEGRINI et al. (2010), entre outros, enfatizaram que o conhecimento de variações na MF nas pastagens é essencial para decisões relacionadas ao manejo do pastejo. A MF pode determinar alteração na taxa de acúmulo de forragem

IPrograma de Pós-graduação em Zootecnia, Universidade Estadual de Maringá (UEM), Campus Universitário, Maringá, PR, Brasil. IDepartamento de Zootecnia, UEM, Campus Universitário, Av. Colombo, 5790, 87020-900, Maringá, PR, Brasil. Email:ccjobim@uem.br. Autor para correspondência.

IIIDepartamento de Plantas Forrageiras e Agrometeorologia, Universidade Federal do Rio Grande do Sul (UFRGS), Porto Alegre, RS, Brasil. 
no pasto (BURNS et al., 1989) e na taxa de consumo de forragem dos animais (BURNS \& SOLLENBERGER, 2002; DIFANTE et al., 2009). O interesse em se medir a MF em pastagens como prática em fazendas que produzem bovinos nos dias de hoje aumentou, para se predizer o desempenho dos animais em pastejo e a produtividade de forragem.

O método considerado como o mais efetivo para estimativas de MF é o método de cortes da forragem, mas, por ser uma técnica destrutiva, apresenta pouca viabilidade prática em razão das amostragens serem bastante trabalhosas (FRAME, 1981). Esse método, evidentemente, na maioria das situações, é inviável, pois requer excessivo dispêndio de tempo, de mão de obra na manipulação de amostras e deve-se considerar que a forragem removida não mais poderá ser consumida por animais (no caso de experimentos avaliados sob pastejo).

Instrumentos não destrutivos e mais expeditos, métodos indiretos, têm sido avaliados visando à utilização em situações de pesquisa e em larga escala em fazendas (JONES \& HAYDOCK, 1970; VARTHA \& MATCHES, 1977; FRAME, 1981). Dentre os métodos indiretos, estão a altura comprimida do relvado, utilizando-se o disco medidor de forragem (DMF) (disk meter) (CASTLE, 1976), denominado por igual de prato ascendente (rising plate meter), a altura não comprimida do dossel com o uso da régua (SHAW et al., 1976), o bastão graduado (BG) (sward stick) (BARTHRAM, 1986), a estimativa visual e o medidor de capacitância (MC) (pasture probe ou sonda eletrônica) (VICKERY et al., 1980).

Os métodos indiretos também exigem o corte de amostras de forragem, porém em números mais reduzido e não necessariamente realizadas em locais de outras avaliações, tornam as amostragens de MF mais funcionais e ágeis, viabilizando a utilização em pastagens com áreas extensas. Os métodos indiretos são os mais indicados para serem utilizados em pastagens com animais em pastejo, permitem amostragens em parcelas experimentais com áreas reduzidas nas quais procedimentos destrutivos poderiam afetar alta proporção da área e interferir em outras avaliações.

Para a calibração dos métodos indiretos, faz-se uso de cortes visando gerar equações de calibração. Após a calibração, as estimativas de MF podem ser usadas para curvas de resposta com equações. Por essa razão, é importante que a calibração possa abranger nos pastos a amplitude de MF (FRAME, 1981; 't MANNEJTE, 2000). No entanto, a maioria das informações com os métodos indiretos provém de pesquisas em forrageiras temperadas.
A literatura internacional relata estudos com os métodos indiretos em que os resultados foram bastante variáveis, em termos de precisão e em diferentes forrageiras utilizadas sob condições de pastejo (GOURLEY \& McGOWAN, 1991; Da SILVA\& CUNHA, 2003; OGURA et al., 2005).

O capim-estrela é uma das mais importantes gramíneas tropicais utilizadas no Sul do Brasil. Essa gramínea é também muito utilizada no mundo em pastagens situadas em regiões tropicais e subtropicais. Todavia, nas condições brasileiras e na literatura internacional, não se encontram disponíveis trabalhos que avaliaram, em pastagens de capim-estrela utilizadas com bovinos, métodos indiretos de estimativas de MF, este é o primeiro estudo dessa natureza.

O objetivo deste estudo foi comparar os métodos indiretos mais utilizados de estimativas de $\mathrm{MF}$ como o $\mathrm{BG}$, o DMF, a régua e o $\mathrm{MC}$ em pastos de capim-estrela utilizados sob condições de pastejo contínuo em distintas alturas do pasto.

\section{MATERIAL E MÉTODOS}

Este estudo foi realizado em área da Fazenda Nossa Senhora Aparecida no Noroeste do Paraná (latitude $23^{\circ} 18^{\prime} \mathrm{S}$, longitude $51^{\circ} 51^{\prime} \mathrm{W}$ e altitude $405,7 \mathrm{~m}$ ), no período de 07/11/2007 a 26/04/2008. O clima da região é o tropical mesotérmico úmido, classificado como $\mathrm{Cfa}$ de acordo com KÖPPEN. As condições de clima foram observadas na Estação de Meteorologia da Universidade Estadual de Maringá, a aproximadamente $19 \mathrm{~km}$ da área experimental (Tabela 1). A temperatura média máxima e a temperatura média mínima foram, respectivamente, de 30,9 e $14,6^{\circ} \mathrm{C}$. A insolação média variou de 5,8 a 8,1 horas dia- ${ }^{-1}$ nos meses de janeiro e março, respectivamente. A precipitação pluviométrica total foi de $1038,8 \mathrm{~mm}$, como médias de concentrações pluviais na região nos meses de novembro, dezembro, janeiro, fevereiro, março e abril, respectivamente, 160; 201,5; 191,6; 153,8; 146,7 e 130,2mm. As condições de clima foram próximas das normalmente observadas na região Noroeste do Paraná. O solo da área é classificado como Latossolo Vermelho Distroférrico Argiloso (Embrapa, 1999), profundo, bem drenado e de textura argilosa (acima de 79\%). A coleta de solo foi realizada a duas profundidades (Tabela 2). Não houve necessidade da aplicação de calcário e de potássio. A adubação de $90 \mathrm{~kg} \mathrm{ha}^{-1} \mathrm{de}_{2} \mathrm{O}_{5}$ (superfosfato simples) foi aplicada em mistura com 2,9 $\mathrm{kg} \mathrm{ha}^{-1}$ de boro (ácido bórico) e zinco (sulfato de zinco) ao solo e em cobertura em 9/11/2007. A adubação nitrogenada no período experimental foi de $250 \mathrm{~kg} \mathrm{ha}^{-1}$ de nitrogênio com nitrato de amônio (33\% de $\mathrm{N})$, em três aplicações, duas com $80 \mathrm{~kg} \mathrm{ha}^{-1}(25 / 11 / 2007$, $15 / 01 / 2008$ ) e uma de $90 \mathrm{~kg} \mathrm{ha}^{-1}$ (15/02/2008). 
Tabela 1 - Temperatura máxima média, temperatura mínima média, temperatura média do ar, precipitação pluviométrica (PP), umidade relativa (UR) do ar e insolação do período experimental.

\begin{tabular}{|c|c|c|c|c|c|c|}
\hline \multirow{3}{*}{ Mês/ano } & \multicolumn{3}{|c|}{ 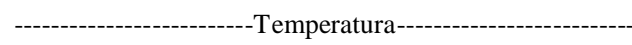 } & \multirow{3}{*}{$\begin{array}{c}\text { PP } \\
\text { Total Mensal } \\
(\mathrm{mm})\end{array}$} & \multirow{3}{*}{$\begin{array}{c}\text { U R } \\
\text { Média } \\
(\%)\end{array}$} & \multirow{3}{*}{$\begin{array}{c}\text { Insolação } \\
\text { Média } \\
\left(\mathrm{h} \mathrm{dia}^{-1}\right)\end{array}$} \\
\hline & Máxima média & Mínima média & Média & & & \\
\hline & 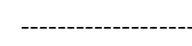 & $---\left({ }^{\circ} \mathrm{C}\right)--$ & 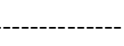 & & & \\
\hline $1 / 11 / 2007$ & 29,9 & 18,4 & 25,0 & 171,4 & 0,64 & 229,0 \\
\hline $1 / 12 / 2007$ & 30,9 & 20,4 & 26,3 & 235,0 & 0,67 & 225,4 \\
\hline $1 / 1 / 2008$ & 29,0 & 20,4 & 25,1 & 117,5 & 0,75 & 181,3 \\
\hline $1 / 2 / 2008$ & 30,0 & 20,4 & 25,2 & 134,6 & 0,75 & 213,2 \\
\hline $1 / 3 / 2008$ & 29,7 & 19,5 & 25,1 & 126,6 & 0,69 & 250,0 \\
\hline $1 / 4 / 2008$ & 27,9 & 17,9 & 23,4 & 172,2 & 0,73 & 215,4 \\
\hline $1 / 5 / 2008$ & 24,3 & 14,6 & 20,2 & 81,5 & 0,68 & 211,0 \\
\hline
\end{tabular}

A área experimental foi estabelecida com capim-estrela em 2004. As pastagens experimentais eram delimitadas por cercas fixas e com áreas aproximadas de 0,9 ha, totalizando a área experimental 7,6ha. Os animais utilizados foram novilhas da raça Nelore com massa corporal inicial média de $270 \mathrm{~kg}$ $( \pm 68 \mathrm{~kg})$.

O delineamento experimental usado foi o inteiramente ao acaso com quatro tratamentos e duas repetições. Os tratamentos consistiram em quatro alturas do pasto: $5,10,15,20 \mathrm{~cm}$. O método de pastejo utilizado foi o pastejo contínuo com taxa de lotação variável (MOTT \& LUCAS, 1952) e a altura do pasto nos piquetes foi regulada por ajustes da taxa de lotação. Os instrumentos utilizados para avaliar a MF foram o $\mathrm{BG}$, a régua, o DMF e o MC. A equipe foi formada por um observador e por três auxiliares que realizaram os cortes da forragem durante todo o período experimental. A altura do pasto foi avaliada por 100 leituras semanais em cada unidade experimental, duas vezes por semana, usando-se o BG e basearam-se no procedimento descrito por BARTHRAM (1986).

Os métodos indiretos de medição de MF foram avaliados em delineamento inteiramente ao acaso com duas repetições. No presente trabalho, o MC foi o modelo Grassmaster II pertencente à marca Speedrite. A régua usada $(50 \mathrm{~cm})$ era de plástico transparente com escala graduada de $1,0 \mathrm{~cm}$. O BG também foi com essa escala de medição com altura de $50 \mathrm{~cm}$ e o DMF usado foi com área de $0,1 \mathrm{~m}^{2}$. Em cada piquete, foram realizados cinco cortes de forragem em áreas escolhidas consideradas representativas da condição média do relvado e altura do pasto preestabelecida para o piquete. Cuidados foram tomados em não contaminar a forragem cortada com solo, com restos de esterco de animais ou com material morto.

Em cada amostra coletada em área demarcada por moldura, foram realizadas, antes do corte da forragem, cinco leituras com o $\mathrm{MC}$, cinco medições com a régua, cinco medições com o BG e, logo após, uma medição com o DMF. Os cortes da forragem foram realizados rente ao solo com tesoura de poda e a área delimitada pela moldura de ferro foi de $0,25 \mathrm{~m}^{2}(50 \mathrm{x} 50 \mathrm{~cm})$. A forragem dos cortes foi colocada em saco de papel, mantida em freezer e posteriormente foi seca em estufa de ventilação forçada de ar por 56 horas a $60^{\circ} \mathrm{C}$. Nas áreas demarcadas pela moldura, o $\mathrm{MC}$ foi posicionado no relvado e automaticamente foi realizada a leitura da MF no visor do instrumento (em $\mathrm{kg}$ de MS ha-1). O BG foi posicionado verticalmente na área demarcada pela moldura com a base de sua haste metálica em contato com o solo e a seguir o marcador móvel plástico foi deslizado até o primeiro contato com a folha mais alta do perfilho, realizando-se então a leitura da altura do pasto. Esse procedimento baseou-se em HODGSON (1990) e foi usado nos cinco locais na área demarcada pela moldura.

A avaliação com o DMF foi realizada posicionando-se a haste metálica verticalmente acima da vegetação da área demarcada pela moldura. A seguir, foi realizada a leitura no contador do DMF e, logo após, o eixo metálico do DMF foi abaixado em direção ao solo. No momento em que a base metálica do DMF entrasse em contato com o solo, esta seria puxada a sua condição inicial, efetuando-se a leitura no aparelho contador do DMF. O valor obtido no aparelho foi calculado subtraindo-se a leitura final do contador inicial.

Os pares de dados de leitura de altura do pasto $\mathrm{x} \mathrm{MF}$, leitura de $\mathrm{MS}$ do $\mathrm{MC} \mathrm{x} \mathrm{MF}$, leitura da régua $x$ MF, leitura do DMF x MF foram tabulados e equações de regressão foram testadas até terceira ordem (PROC REG; pacote estatístico SAS, 2002). A equação de regressão escolhida foi a que apresentou mais alta significância, mais alto coeficiente de determinação $\left(\mathrm{r}^{2}\right)$ e menor coeficiente de variação (CV). 
Tabela 2 - Características químicas no solo da área experimental.

\begin{tabular}{lcccccccc}
\hline $\begin{array}{l}\text { Profundidade } \\
(\mathrm{cm})\end{array}$ & $\mathrm{pH} \mathrm{CaCl}_{2}$ & $\begin{array}{c}\mathrm{C} \\
\mathrm{g} \mathrm{dm}^{-3}\end{array}$ & $-\mathrm{H}+\mathrm{Al}$ & $\mathrm{Ca}+2$ & $\mathrm{Ca}+\mathrm{Mg}$ & $\mathrm{K}+$ & $\begin{array}{c}\mathrm{P} \\
\mathrm{mg} \mathrm{dm}^{3}\end{array}$ \\
\hline $0-5$ & 5,4 & 30,2 & 5,1 & 6,9 & 10,2 & 0,78 & 22,4 & 3,8 \\
$\mathrm{mg} \mathrm{kg}^{-1}$ & \\
$5-15$ & 5,3 & 25,1 & 5,2 & 6,6 & 9,6 & 0,73 & 9,4 & 3,8 \\
\hline
\end{tabular}

\section{RESULTADOS E DISCUSSÃO}

$\mathrm{O}$ BG, DMF e a régua apresentaram coeficientes de determinação considerados baixos (Tabela 3). O MC teve baixa capacidade de avaliação de MF. Os coeficientes de variação com o BG, DMF e a régua foram altos. As regressões lineares apresentaram coeficientes de determinação significativos $(\mathrm{P}<0,05)$ para as relações da MF com o DMF e com a régua. Conforme a equação de regressão, as médias de MF para o $\mathrm{BG}$, o DMF e régua nas alturas do pasto de 5,10 , 15 e $20 \mathrm{~cm}$ foram, respectivamente, $2793,3069,3728 \mathrm{e}$ $4771 \mathrm{~kg}$ de $\mathrm{MS} \mathrm{ha}^{-1}$; de 2081, 2936, 3791 e 4646kg de MS $\mathrm{ha}^{-1} \mathrm{e}$ de 1947, 2902, 3866 e 4826kg de MS ha' ${ }^{-1}$.

Verifica-se que, conforme a equação de regressão, o BG nos pastos com altura do pasto de cinco $\mathrm{cm}$ os valores de MF foram mais altos em comparação ao DMF e à régua. Nas alturas do pasto de 15 e $20 \mathrm{~cm}$, os valores obtidos por cálculo usandose as equações de regressão geradas com os métodos do BG, do DMFe com a régua foram próximos. Os baixos valores para a predição da MF nas pastagens de capimestrela observados com o $\mathrm{BG}$, o DMF e a régua podem estar relacionados principalmente aos efeitos da estação do ano, das estruturas de plantas, ao hábito de pastejo das novilhas e à intensidade de utilização pelos animais de determinadas áreas (patchs) no pasto. Neste estudo, cabe considerar que não houve o efeito de observador. Sabe-se que, à medida que ocorre o avanço na estação de crescimento, aumenta o teor de MS nos perfilhos em razão do alongamento dos entrenós nos colmos lignificados, que causam o aumento no teor de MS e alterações na estrutura e no arranjo espacial de folhas. Outros fatores, que não foram mensurados, podem também ter contribuído para a relativa baixa precisão dos métodos $\mathrm{BG}$, $\mathrm{DMF}$ e régua, como o relevo do solo e as variabilidades espacial na MF, na massa individual e no número de perfilhos. Esses fatores, descritos na literatura, são típicos e verificados com maior frequência em situações de pastagens mantidas sob pastejo (VALLENTINE, 1990; HIRATA, 2000; OGURA et al., 2005).

No presente estudo, as áreas mais próximas dos reservatórios de água, por volta de $18 \%$ das áreas dos piquetes, visualmente, apresentavam-se com maior altura do pasto em comparação à média e, muito provavelmente, com maior MF. Essas áreas permaneceram com maior concentração de animais ao longo do dia, tendo-se em consequência o efeito residual da maior deposição de fezes e urinas dos anos e este efeito pode estar também relacionado à baixa precisão obtida com o $\mathrm{BG}, \mathrm{DMF}$ e a régua.

Em pastagens que se encontram com fertilidade alta no solo, com MF apropriadas e usadas por animais em pastejo, normalmente se verifica maior deposição de nutrientes pela excreta de fezes e urina dos animais ao longo do tempo nas áreas próximas de aguadas e que são mais utilizadas para o ócio e a ruminação. Entretanto, BRAGA et al. (2009), em pastos de capim-marandu [Brachiaria brizantha (A. Rich.) Stapf cv. 'Marandu'] mencionaram que os modelos gerados de estimativa de MF com o DMF e o BG, com base em mensurações de altura do pasto, os valores de intercepto e de coeficiente angular são especialmente influenciados pelos meses do período experimental. Os autores sugerem que modelos específicos gerados para cada mês podem proporcionar melhorias de predição de massa de forragem, em comparação ao modelo que abrange o total da estação.

Tabela 3 - Coeficiente de correlação (r), nível de probabilidade (Pr), coeficiente de determinação $\left(\mathrm{R}^{2}\right)$, coeficiente de variação (CV) e equação de regressão obtida em pastos de capim-estrela com métodos de avaliação de massa de forragem (BG - bastão graduado, DMF - disco medidor de forragem, MC - Medidor de capacitância, RG - régua), expressa em kg de MS/ha.

\begin{tabular}{lllllll}
\hline Método & $\mathrm{r}$ & \multicolumn{1}{c}{$\operatorname{Pr}$} & $\mathrm{R}^{2}$ & $\mathrm{Pr}$ & \multicolumn{1}{c}{ CV\% } & \multicolumn{1}{c}{ Equação } \\
\hline BG & 0,54 & 0,001 & 0,56 & 0,001 & 66,39 & $\mathrm{Y}=2902,1-60,15 \mathrm{x}+7,68 \mathrm{x}^{2}$ \\
DMF & 0,63 & 0,001 & 0,64 & 0,001 & 54,80 & $\mathrm{Y}=1226,64+170,97 \mathrm{x}$ \\
MC & $\mathrm{ns}$ & $\mathrm{ns}$ & $\mathrm{ns}$ & $\mathrm{ns}$ & $\mathrm{ns}$ & \\
RG & 0,55 & 0,001 & 0,56 & 0,001 & 61,92 & $\mathrm{Y}=987,02+191,95 \mathrm{x}$ \\
\hline
\end{tabular}

ns = não significativo

Ciência Rural, v.41, n.11, nov, 2011. 
Diferenças alométricas entre as estruturas de plantas, nas áreas utilizadas nas medições com o DMF, podem ter reduzido o valor de predição desse instrumento no presente trabalho. Segundo FEHMI \& STEVENS (2009), a correlação entre a altura do pasto do DMF com a MF é dependente de uma relação consistente entre a densidade de perfilho, a resistência à compressão (resiliência) e a altura das plantas, sendo que diferenças entre esses fatores podem determinar a baixa capacidade de predizer a MF. Com relação ao MC, atribui-se a alta participação de colmos, especialmente nos pastos mantidos com as alturas do pasto mais altas e isso pode ter influenciado seus parâmetros de predição, determinando sua inadequação neste trabalho. Segundo GONZALEZ et al. (1990), em pastos com MF superior a 7000kg de MS $\mathrm{ha}^{-1}$, verifica-se, frequentemente, o aumento na variabilidade dos valores obtidos em razão da alta proporção de colmos. JONES et al. (1977) relataram que é alta a probabilidade de insucesso do MC em predizer a MF de pastos de gramíneas tropicais utilizados sob pastejo. OGURA et al. (2005) observaram alta predição da quantidade de MF usando o MC. Entretanto, os autores evidenciaram que, em pastos de [Paspalum notatum Flügge] mantidos com alta MF, a capacidade de avaliação do MC foi baixa. A utilização do $\mathrm{MC}$ em pastos de gramíneas tropicais que apresentam hábito de crescimento estolonífero mantidos com MF superiores a $3700 \mathrm{~kg}$ de MS ha ${ }^{-1}$ necessita de mais estudos.

DA SILVA \& CUNHA (2003) avaliaram o DMF e o BG em cultivares do gênero Cynodon ('Tifton85', 'Coastcross-1' e 'Florakirk') utilizadas sob regime de pastejo contínuo com as alturas do pasto de 5, 10, 15 e $20 \mathrm{~cm}$ e obtiveram, ao longo do período experimental, valores de coeficiente de determinação para o DMF na primavera e verão com as cultivares 'Tifton-85', 'Coastcross-1' e 'Florakirk', respectivamente, de 0,73 e 0,$77 ; 0,49$ e 0,86 e de 0,54 e 0,80 . Os autores reportaram que os coeficientes de variação com a régua nas estações da primavera e verão com as cultivares 'Tifton-85', 'Coastcross-1' e 'Florakirk' foram, respectivamente, 0,72 e 0,$81 ; 0,40 \mathrm{e}$ 0,85 e de 0,60 e 0,77 . Segundo os autores, as diferenças morfológicas e de arquitetura entre as cultivares e alturas do pasto contribuíram para os baixos valores de intercepto e de coeficiente angular da equação de regressão com o DMF, em comparação aos obtidos com a régua.

CAUDURO et al. (2006), em pastos de azevém [Lolium multiflorum Lam.] sob pastoreio rotativo e duas intensidades de pastejo, avaliaram o DMF, o BG e o MC e verificaram também baixa precisão com os métodos. Em pastos de Festuca arundinaceae
Schreb, HARMONEY et al. (1997) observaram valores de coeficiente de determinação de 0,08 com o BG e 0,85 com o DMF. BRANSBY et al. (1977) e VARTHA \& MATCHES (1997) obtiveram valores de coeficiente de determinação entre 0,09 e 0,94 para o DMF igualmente em pastos de Festuca arundinaceae. BANDINELLI et al. (2003) avaliaram a eficiência do BG e do DMF em pastagens consorciadas de aveia e azevém e constataram valores de coeficiente de determinação para o BG de 0,506 e para o DMF de 0,506. BRAGA et al. (2009), avaliando pastagens de capim-marandu, com pastejo rotativo, obtiveram coeficientes de determinação de 0,82 e 0,91 para o disco e a régua, respectivamente.

OGURA et al. (2005) avaliaram o BG, oDMF e o MC em pastos de Paspalum notatum e de Eremochloa ophiuroides. Nos pastos de Paspalum notatum, nas situações de alta MF com alta proporção de colmos, os métodos apresentaram baixa precisão. Da SILVA \& CUNHA (2003) avaliaram o DMF e a régua em pastos das cultivares de Cynodon spp. 'Florakirk', 'Tifton 85' e 'Coastcross-1' e reportaram que a calibração dos métodos deve ser frequente, para melhor precisão nas equações geradas e realizadas por cultivar e por estação do ano.

Neste estudo, os métodos avaliados mostraram-se mais rápidos, com economia de mão de obra comprovando viabilidade de utilizações em pesquisa com pastagens e, evidentemente, devem ser preconizados alertando-se sobre as suas restrições para o monitoramento da MF em pastagen s de capim-estrela usadas por novilhas sob condições de pastejo contínuo. Os valores com os métodos neste trabalho não atingiram a precisão preconizada por THOMPSON (1986), que mencionou que valores de coeficiente de determinação inferior a 0,75 não são satisfatórios.

\section{CONCLUSÃO}

Medições de massa de forragem em pastos de capim-estrela mantidos sob pastejo contínuo com bovinos e com a amplitude de altura de manejo do pasto entre cinco e $20 \mathrm{~cm}$ devem ser realizadas preferivelmente com o disco medidor de forragem, ou ainda com o bastão graduado e a régua.

\section{REFERÊNCIAS}

BANDINELLI, D.G. et al. Variáveis morfogênicas de Andropogon lateralis Nees submetido a níveis de nitrogênio nas quatro estações do ano. Ciência Rural, v.33, n.1, p.71-76, 2003. Disponível em: <http://www.scielo.br/ scielo.php?script=sci_arttext\&pid=S01034782003000100011\&lng $=$ pt $\&$ nrm=iso $>$. Acesso em: 25 dez. 2010. doi: 10.1590/ S0103-84782003000100010. 
BARTHRAM, G.T. Experimental techniques: the HFRO sward stick. Penicuik: Hill Farming Research Organization, 1986. p.29-30. (Biennal Report 1984-1985).

BENKOBI, L. et al. Protocol for monitoring standing crop in grasslands using visual obstruction. Journal of Range Management, v.53, p.627-633, 2000.

BRAGA, G.J, et al. Quantifying herbage mass on rotationally stocked palisadegrass pasture using indirect methods. Scientia Agricola, v.66, n.1, p.127-131, 2009. Disponível em:<http:/ $/ \mathrm{www}$. scielo.br/scielo.php?script=sci_arttext\&pid=S010390162009000100018\&lng=pt\&nrm=iso $>$. Acesso em: $18 \mathrm{dez}$. 2010. doi: 10.1590/S0103-90162009000100018.

BRANSBY, D.I. et al. Disk meter for rapid estimation of herbage yield in grazing trials. Agronomy Journal, v.69, n.3, p.393-396, 1977.

BURNS, J.C. et al. The relationship of herbage mass and characteristics to animal responses in grazing experiments. In: MARTEN, G.C. Grazing research: design, methodology, and analysis. Madison: Crop Science Society of America, American Society of Agronomy, 1989. Cap.2, p.7-19.

BURNS, J.C.; SOLLENBERGER, L.E. Grazing behavior of ruminants and daily performance from warm-season grasses. Crop Science, v.42, n.3, p.873-881, 2002.

CASTLE, M.E. A simple disc instrument for estimating herbage yield. Journal of the British Grassland Society, v.31, n.2, p.37-40, 1976 .

CAUDURO, G.F. et al. Comparação de métodos de medida indireta de massa de forragem em pasto de azevém anual (Lolium multiflorum Lam.). Ciência Rural, v.36, n.5, p.1617-1623, 2006. Disponível em: <http://www.scielo.br/ scielo.php ? script=sci_arttext \& pid=S 0103 $84782006000500044 \& \operatorname{lng}=$ pt\&nrm=iso $>$. Acesso em: 25 dez. 2010. doi: 10.1590/S0103-84782006000500043.

DA SILVA, S.C.; CUNHA, W.F. Métodos indiretos para estimar a massa de forragem em pastos de Cynodon spp. Pesquisa Agropecuária Brasileira, v. 38 , n. 8 , p.981989, 2003. Disponível em: <http://www.scielo.br/ scielo.php? script $=\mathrm{sci}_{-}$art text \& pid $=\mathrm{S} 0100-$ $204 X 2003000800011 \& \operatorname{lng}=$ pt\&nrm=iso $>$. Acesso em: 25 dez. 2010. doi: 10.1590/S0100-204X2003000800011.

DIFANTE, G.S. et al. Sward structure and nutritive value of tanzania guineagrass subjected to rotational stocking managements. Revista Brasileira de Zootecnia, v.38, n.1, p.9-19, 2009. Disponível em: <http://www.scielo.br/ scielo.php? script=sci_arttext\&pid=S 1516 $35982009000100002 \& \operatorname{lng}=\mathrm{pt} \& \mathrm{nrm}=\mathrm{iso}>$. Acesso em: $25 \mathrm{dez}$. 2010. doi: $10.1590 / \mathrm{S} 1516-35982009000100001$.

EMBRAPA. Sistema brasileiro de classificação de solos. Brasília: Empresa Brasileira de Pesquisa Agropecuária, 1999. 412p.

FEHMI, J.S.; STEVENS, J.M. A plate meter inadequately estimated herbage mass in a semi-arid grassland. Grass and Forage Science, v.64, n.3, p.322-327, 2009.

FORAGE AND GRAZING TERMINOLOGY COMMITTEE. Terminology for grazing lands and grazing animals. Journal of Production Agriculture, v.5, n.2, p.191-201, 1992.

FRAME, J. Herbage mass. In: HODGSON, J. et al. Sward measurement handbook. Berkshire: Bristh Grassland Society, 1981. Cap.3, p.39-67.
GOURLEY C.J.P., McGOWAN A.A. Assessing differences in pasture mass with an automated rising plate meter and a direct harvesting technique. Australian Journal of Experimental Agriculture and Animal Husbandry, v.31, n.3, p.337-339, 1991.

GONZALEZ, M.A. et al. Plant height, disk, and capacitance meters used to estimate Bermudagrass herbage mass. Agronomy Journal, v.82, n.5, p.861-864, 1990.

HARMONEY, K.R. et al. Determination of pasture mass using four indirect methods. Agronomy Journal, v.89, n.2, p.665672, 1997.

HIRATA, M. Quantifying spatial heterogeneity in herbage mass and consumption in pastures. Journal of Range Management, v.53, p.315-321, 2000.

HODGSON, J. Nomenclature and definitions in grazing studies. Grass and Forage Science, v.34, n.1, p.11-17, 1979.

HODGSON, J. Grazing management: science into practice. Essex: Longman Scientific \& Technical, 1990. 203p.

JONES, R.J.; HAYDOCK, K.P. Yield estimation of tropical and temperate pasture species using an electronic pasture meter. Journal of Agriculture Science, v.75, n.1, p.27-36, 1970.

JONES, R.M. et al. Limitations of the electronic capacitance meter in measuring yields of grazed tropical pastures. Journal of the British Grassland Society, v.32, n.2, p.105-113, 1977.

MOTT, G.O.; LUCAS, H.L. The design, conduct and interpretation of grazing trials on cultivated and improved pastures. In: INTERNATIONAL GRASSLAND CONGRESS, 6., 1952, Pennsylvania. Proceedings... Pennsylvania: State College, 1952. p.1380-1385.

OGURA, S. et al. Estimation of herbage mass in a bahia grass (Paspalum notatum) and a centipede grass (Eremochloa ophiuroides) pasture using a capacitance probe, a sward stick and a rising plate. Tropical Grasslands, v.39, n.1, p.22-30, 2005.

PELLEGRINI, C.B. et al. Precisão da estimativa da massa de forragem com discos medidores em pastagem nativa. Ciência Rural, v.40, n.1, p.163-169, 2010. Disponível em:<http:// www.scielo.br/scielo.php? script $=$ sci_arttext $\&$ pid $=$ S0 103 $84782010000100026 \& \operatorname{lng}=\mathrm{pt} \& \mathrm{nrm}=\mathrm{iso}>$. Acesso em: $25 \mathrm{dez}$. 2010. doi: 10.1590/S0103-84782010000100026.

SHAW, N.H. et al. Pasture measurements. In: SHAW, N.H.; BRYAN, W.W. (Ed.). Tropical Pasture Research. Hurley: Commonwealth Bureau of Pastures and Field Crops, 1976. Cap.10, p.235-250.

't MANNEJTE, L. Measuring biomass of grassland vegetation. In: 't MANNEJTE, L.; JONES, R.M. (Ed.). Field and laboratory methods for grassland and animal production research. Wallingford: CAB International, 2000. Cap.7, p.151-177.

THOMPSON, J R. Effect of grazing on infiltration in a western watershed. Journal of the Soil and Water Conservation, v.23, n.2, p.63-65, 1968.

VALLENTINE, J.F. Grazing management. San Diego: Academic, 1990. 533p.

VARTHA, E.W.; MATCHES, A.G. Use of a weighted disk measure as an aid in sampling the herbage yield on tall fescue pastures grazed by cattle. Agronomy Journal, v.69, n.5, p.888-890, 1977.

VICKERY, P.J. et al. An improved electronic capacitance meter for estimating herbage mass. Grass and Forage Science, v.35, n.3, p.247-252, 1980. 